\title{
DIRAC MONOPOLES IN THE ERNST-SCHWARZSCHILD SPACETIME
}

\author{
A. A. BYTSENKO ${ }^{1}$ AND YU. P. GONCHAROV ${ }^{2}$ \\ 1. Departamento de Fisica, Universidade Estadual de Londrina \\ Caixa Postal 6001, Londrina-Parana, Brazil \\ 2. Theoretical Group, Experimental Physics Department, State Polytechnical University \\ Sankt-Petersburg 195251, Russia
}

November 7, 2018

\begin{abstract}
It is discussed that the Ernst-Schwarzschild metric describing a nonrotating black hole in the external magnetic field admits the solutions of the Dirac monopole types for the corresponding Maxwell equations. The given solutions are obtained in explicit form and a possible influence of the conforming Dirac monopoles on Hawking radiation is also outlined.
\end{abstract}

\section{Introduction}

In astrophysics for a long time the physics of black holes immersed into an external magnetic field has been studied ( see, e.g., the review in Ref. 1). In view of it the whole class of solutions of the Einstein-Maxwell equations was found to model a black hole in an external electromagnetic field. Referring for more details to Refs. 3 we should like here to notice that an isolated black hole might possess the internal magnetic fields of the Dirac monopole types. The latter configurations should be connected with nontrivial topological properties of black holes and could have an essential influence on quantum processes near black holes, for instance, on Hawking radiation. A number of examples of such configurations may be found in Refs. 3 and references therein. Physically, the existence of those configurations should be obliged to the natural presence of magnetic $\mathrm{U}(\mathrm{N})$-monopoles (with $N \geq 1$ ) on black holes though the total (internal) magnetic charge (abelian or nonabelian) of black hole remains equal to zero. One can consider that monopoles reside in black holes as quantum objects without having influence on the black hole metrics. They could reside in the form of monopole gas in which the process of permanent creation and annihilation of the virtual monopole-antimonopole pairs occurs so that the summed internal magnetic charge (i. e., related with topological properties) is equal to zero while the external one (not connected with topological properties) may differ from zero 
( e. g. on the Reissner-Nordström or, more generally, Kerr-Newman black holes with magnetic charges). While existing the virtual monopole-antimonopole pair can interact with a particle and, by this, increasing the Hawking radiation (see Refs. 3 and references therein).

There arises the question whether there exist the Dirac-like monopole configurations on the black holes immersed into an external magnetic field. Within the given note we shall show that the answer is affirmative by example of the ErnstSchwarzschild spacetime [4] describing Schwarzschild black hole in asymptotically homogeneous magnetic field. The metric of spacetime manifold in question is

$$
d s^{2}=g_{\mu \nu} d x^{\mu} \otimes d x^{\nu} \equiv \Lambda^{2}\left(a d t^{2}-a^{-1} d r^{2}-r^{2} d \vartheta^{2}\right)-\frac{r^{2} \sin ^{2} \vartheta d \varphi^{2}}{\Lambda^{2}}
$$

with $a=1-2 M / r, \Lambda=1+\frac{1}{4} B^{2} r^{2} \sin ^{2} \vartheta,|g|=\left|\operatorname{det}\left(g_{\mu \nu}\right)\right|=\left(\Lambda^{2} r^{2} \sin \vartheta\right)^{2}$ and $0 \leq r<\infty, 0 \leq \vartheta<\pi, 0 \leq \varphi<2 \pi$. At this the surface $t=$ const, $r=$ const is an ellipsoid with topology $\mathcal{S}^{2}$.

Throughout the paper we employ the system of units with $\hbar=c=G=1$, unless explicitly stated.

\section{Dirac Monopole Type Solutions}

To write down the Maxwell equations in spacetime with metric (1) we need to know the action of the Hodge star operator ${ }^{*}$ on 2 -forms $F=F_{\mu \nu} d x^{\mu} \wedge d x^{\nu}$ which is defined for any $k$-dimensional (pseudo)riemannian manifold $B$ provided with a (pseudo)riemannian metric $g_{\mu \nu}$ by the relation (see, e. g., Refs. 5)

$$
F \wedge * F=\left(g^{\mu \alpha} g^{\nu \beta}-g^{\mu \beta} g^{\nu \alpha}\right) F_{\mu \nu}^{a} F_{\alpha \beta}^{a} \sqrt{|g|} d x^{1} \wedge d x^{2} \cdots \wedge d x^{k}
$$

in local coordinates $x^{\mu}$. In the case of the metric (1) this yields for the basis elements

$$
\begin{gathered}
*(d t \wedge d r)=\sqrt{|g|} g^{t t} g^{r r} d \vartheta \wedge d \varphi=-\frac{r^{2} \sin \vartheta}{\Lambda^{2}} d \vartheta \wedge d \varphi \\
*(d t \wedge d \vartheta)=-\sqrt{|g|} g^{t t} g^{\vartheta \vartheta} d r \wedge d \varphi=\frac{\sin \vartheta}{a \Lambda^{2}} d r \wedge d \varphi \\
*(d t \wedge d \varphi)=\sqrt{|g|} g^{t t} g^{\varphi \varphi} d r \wedge d \vartheta=-\frac{\Lambda^{2}}{a \sin \vartheta} d r \wedge d \vartheta \\
*(d r \wedge d \vartheta)=\sqrt{|g|} g^{r r} g^{\vartheta \vartheta} d t \wedge d \varphi=\frac{a \sin \vartheta}{\Lambda^{2}} d t \wedge d \varphi \\
*(d r \wedge d \varphi)=-\sqrt{|g|} g^{r r} g^{\varphi \varphi} d t \wedge d \vartheta=-\frac{a \Lambda^{2}}{\sin \vartheta} d t \wedge d \vartheta \\
*(d \vartheta \wedge d \varphi)=\sqrt{|g|} g^{\vartheta \vartheta} g^{\varphi \varphi} d t \wedge d r=\frac{\Lambda^{2}}{r^{2} \sin \vartheta} d t \wedge d r
\end{gathered}
$$

so that $*^{2}=* *=-1$, as should be for the manifolds with lorentzian signature [5]. 
The Maxwell equations are

$$
\begin{gathered}
d F=0, \\
d * F=0
\end{gathered}
$$

for electromagnetic vector-potential $A=A_{\mu} d x^{\mu}, F=d A$ with the exterior differential $d=\partial_{t} d t+\partial_{r} d r+\partial_{\vartheta} d \vartheta+\partial_{\varphi} d \varphi$ in coordinates $t, r, \vartheta, \varphi$. It is clear that (4) is identically satisfied (the Bianchi identity) so that it is necessary to solve only the Eq. (5). Let us search for $A$ in the form $A=A_{\varphi}(r, \vartheta) d \varphi$, i.e., putting the components $A_{t}=A_{r}=A_{\vartheta}=0$. This entails $F=d A=$ $\partial_{r} A_{\varphi} d r \wedge d \varphi+\partial_{\vartheta} A_{\varphi} d \vartheta \wedge d \varphi$ and with the help of (3)

$$
* F=-\frac{a \Lambda^{2}}{\sin \vartheta} \partial_{r} A_{\varphi} d t \wedge d \vartheta+\frac{\Lambda^{2}}{r^{2} \sin \vartheta} \partial_{\vartheta} A_{\varphi} d t \wedge d r .
$$

Then the Eq. (5) take the form

$$
\begin{gathered}
\frac{\partial}{\partial r}\left[\sqrt{|g|}\left(g^{r r} g^{\varphi \varphi} \frac{\partial A_{\varphi}}{\partial r}\right)\right]+\frac{\partial}{\partial \vartheta}\left[\sqrt{|g|}\left(g^{\vartheta \vartheta} g^{\varphi \varphi} \frac{\partial A_{\varphi}}{\partial \vartheta}\right)\right]= \\
\frac{\partial}{\partial r}\left[\frac{a \Lambda^{2}}{\sin \vartheta} \frac{\partial A_{\varphi}}{\partial r}\right]+\frac{\partial}{\partial \vartheta}\left[\frac{\Lambda^{2}}{r^{2} \sin \vartheta} \frac{\partial A_{\varphi}}{\partial \vartheta}\right]=0 .
\end{gathered}
$$

Now we employ the ansatz $A_{\varphi}=-\alpha f(\vartheta) / \Lambda$ with some constant $\alpha$ and inserting it into (7) entails the equation for function $f(\vartheta)$

$$
\sin \vartheta \frac{d^{2} f}{d^{2} \vartheta}-\cos \vartheta \frac{d f}{d \vartheta}=0
$$

The solution of (8) necessary to us is $f(\vartheta)=\cos \vartheta$ so

$$
A=-\frac{\alpha \cos \vartheta}{\Lambda} d \varphi
$$

To fix the constant $\alpha$ let us require the fulfillment the Dirac charge quantization condition

$$
\int_{\mathcal{S}^{2}} F=\int_{\mathcal{S}^{2}} \partial_{\vartheta} A_{\varphi} d \vartheta \wedge d \varphi=4 \pi q=4 \pi \frac{n}{e}
$$

with magnetic charge $q=n / e, n \in \mathcal{Z}$, the set of integers, where we integrate over any surface $t=$ const, $r=$ const with topology $\mathcal{S}^{2}, e$ is elementary electric charge. The direct evaluation gives

$$
\int_{\mathcal{S}^{2}} F=2 \pi \alpha \int_{0}^{\pi} \frac{\sin \vartheta}{\Lambda}\left(1+\frac{B^{2} r^{2} \cos ^{2} \vartheta}{2 \Lambda}\right) d \vartheta=4 \pi \alpha,
$$

so that $\alpha=n / e$. Also it is not complicated to check that the Gauss theorem holds true

$$
\int_{\mathcal{S}^{2}} * F=0
$$


One can notice that relation (9) passes on to the conforming one for the case of the pure Schwarzschild metric, i.e. at $B=0[\underline{3}$. Finally it is easy to check that the given solutions satisfy the Lorentz gauge condition that can be written in the form $\operatorname{div}(A)=0$, where the divergence of 1 -form $A=A_{\mu} d x^{\mu}$ is defined by the relation $\operatorname{div}(A)=\frac{1}{\sqrt{|\delta|}} \partial_{\mu}\left(\sqrt{|\delta|} g^{\mu \nu} A_{\nu}\right)$.

\section{Concluding Remarks}

Mathematical reason for existence of the solutions obtained is the following one. It should be noted that the standard spacetime topology on which the metric (1) with arbitrary $a=a(r)$ can be realized in a natural way is of bhform. As was discussed in Refs. 3, such topology admits countable number of complex line bundles while each complex line bundle $E$ can be characterized by its Chern number $n \in \mathcal{Z}$. The solutions obtained are just connections in the mentioned bundles - Dirac monopoles. But it should be emphasized that the total (internal) magnetic charge $Q_{m}$ of system(black hole + external magnetic field) which should be considered as the one summed up over all the monopoles remains equal to zero because

$$
Q_{m}=\frac{1}{e} \sum_{n \in \mathcal{Z}} n=0
$$

so the external observer does not see any magnetic charge of system though the monopoles are present in the sense described above.

On the other hand, the nontrivial topological properties of spacetimes may play essential role while studying quantum geometry of fields on them (see, e. g., our reviews [6]). Therefore physically the results obtained could mean that in the given spacetime there exist topologically inequivalent configurations (TICs) for various fields. Each TIC corresponds to its Chern number $n \in \mathcal{Z}$. TIC with $n=0$ can be called untwisted, while the rest of the TICs with $n \neq 0$ should be referred to as twisted. For example, TICs of complex scalar field $\phi$ with mass $\mu_{0}$ should obey the equations

$$
|g|^{-1 / 2}\left(\partial_{\mu}-i e A_{\mu}\right)\left[g^{\mu \nu}(|g|)^{1 / 2}\left(\partial_{\nu}-i e A_{\nu}\right) \phi\right]=-\mu_{0}^{2} \phi,
$$

where along with external electromagnetic field $A=-B^{2} r^{2} \sin ^{2} \vartheta /(2 \Lambda) d \varphi$ we should include the addendum corresponding to (9) so that the full $A$ of (14) would be of the form

$$
A=-\left(\frac{B^{2} r^{2} \sin ^{2} \vartheta}{2 \Lambda}+\frac{n \cos \vartheta}{e \Lambda}\right) d \varphi
$$

Analogously this also holds true for spinor field.

Under the circumstances one can speak about the Hawking radiation process for any TIC of complex scalar or spinor fields and one may try to get the luminosity $L(n)$ with respect to the Hawking radiation for TIC with the Chern 
number $n$. We can interpret $L(n)$ with $n \neq 0$ as an additional contribution to the Hawking radiation due to the additional charged particles leaving the black hole because of the interaction with monopoles and the conforming radiation can be called the monopole Hawking radiation [7. Under this situation, for the all configurations luminosity $L$ of black hole in question with respect to the Hawking radiation to be obtained, one should sum up over all $n$, i. e.

$$
L=\sum_{n \in \mathcal{Z}} L(n) \text {. }
$$

As a result, we can expect a marked increase of Hawking radiation from black holes under consideration. The above program has been to a large extent realized for Schwarzschild black holes [3, 8] and there is an interest to look at how the results obtained before would be changed in the presence of external magnetic field.

But for to get an exact value of this increase one should apply numerical methods. In the case of the pure Schwarzschild black hole, for example, it was found that the contribution due to monopoles can be of order $11 \%$ of the total pion-kaon luminosity [3] while it is of order $22 \%$ for electron-positron case [8]. So that it would be interesting enough to evaluate similar increase in the case of the Ernst-Schwarzschild metric.

\section{Acknowledgements}

The work of authors was supported in part by the Russian Foundation for Basic Research (grant No. 01-02-17157).

\section{References}

[1] A. N. Aliev and D. V. Gal'tsov, Uspehi. Fiz. Nauk 157, 129 (1989).

[2] D. V. Gal'tsov, Particles and Fields in the Vicinity of Black Holes (Moscow University Press, Moscow, 1986).

[3] Yu. P. Goncharov and N. E. Firsova, Int. J. Mod. Phys. D 5, 419 (1996); Nucl. Phys. B 486, 371 (1997); Phys. Lett. B 478, 439 (2000).

[4] F. J. Ernst, J. Math. Phys. 17, 54 (1976).

[5] A. L. Besse, Einstein Manifolds (Springer-Verlag, Berlin, 1987);

M. M. Postnikov, Riemannian Geometry (Factorial, Moscow, 1998).

[6] Yu. P. Goncharov, Int. J. Mod. Phys. A 9, 1 (1994);

A. A. Bytsenko, G. Cognola, L. Vanzo and S. Zerbini, Phys. Rep. 266, 1 (1996).

[7] Yu. P. Goncharov, Pis'ma v ZhETF 69, 619 (1999);

Phys. Lett. B 458, 29 (1999).

[8] Yu. P. Goncharov and N. E. Firsova, Mod. Phys. Lett. A 16, 2399 (2001). 